TAUP-2136-94

\title{
Galilean Limit of Equilibrium Relativistic Mass Distribution for Indistinguishable Events
}

\author{
L. Burakovsky* and L.P. Horwitz ${ }^{\dagger}$ \\ School of Physics and Astronomy \\ Raymond and Beverly Sackler Faculty of Exact Sciences \\ Tel-Aviv University, Tel-Aviv 69978, ISRAEL
}

\begin{abstract}
The relativistic distribution for indistinguishable events is considered in the mass-shell limit $m^{2} \cong M^{2}$, where $M$ is a given intrinsic property of the events. The characteristic thermodynamic quantities are calculated and subject to the zero-mass and the high-temperature limits. The results are shown to be in agreement with the corresponding expressions of an on-mass-shell relativistic kinetic theory. The Galilean limit $c \rightarrow \infty$, which coincides in form with the low-temperature limit, is considered. The theory is shown to pass over to a nonrelativistic statistical mechanics of indistinguishable particles.
\end{abstract}

Key words: special relativity, relativistic Jüttner-Synge, mass-shell limit, Galilean limit.

PACS: 03.30.+p, 05.20.Gg, 05.30.Ch, 98.20.-d

\footnotetext{
*Bitnet: BURAKOV@TAUNIVM.TAU.AC.IL.

${ }^{\dagger}$ Bitnet: HORWITZ@TAUNIVM.TAU.AC.IL. Also at Department of Physics, Bar-Ilan University, Ramat-Gan, Israel
} 


\section{Introduction}

In this paper we consider the mass-shell and the Galilean limits of equilibrium relativistic distribution for indistinguishable events studied in a previous work [1]. In that work we studied an identical many-body system within the framework of a manifestly covariant relativistic statistical mechanics discussed in a series of papers [2]-[4]. In this framework, for an $N$-body system, the $N$ events generating the $N$ particle world lines are considered as the fundamental dynamical objects of the theory; they are characterized by positions $q^{\mu}=(c t, \mathbf{q})$ and energy-momenta $p^{\mu}=(E / c, \mathbf{p})$ in an $8 N$-dimensional phase space. Their motion is parametrized by a continuous Poincaréinvariant parameter called the historical time. Such a system of $N$ events is described by generalized Boltzmann equation [5], whose equilibrium solution gives the distribution functions (for both bosonic and fermionic events treated simultaneously in one expression) coinciding with the corresponding grand canonical distributions obtained in ref. [2] for the static Gibbs ensembles. Upon integration of this distribution function over angular and hyperangular variables, one obtains the relativistic mass distribution [1]. We found expressions for the pressure and the energy density in such a system and obtained the relativistic equation of state. Now we turn to a mass-shell form of that equilibrium distribution and to its Galilean (nonrelativistic) limit.

The Galilean limit of a manifestly covariant relativistic statistical mechanics was considered in refs. [6], [7], by taking $c \rightarrow \infty$ (compared to all other velocities). In this limit the relativistic relation between the energy $E$ and the mass $m$

$$
E^{2}=m^{2}+\mathbf{p}^{2}
$$

transforms to

$$
E=m+\frac{\mathbf{p}^{2}}{2 m}
$$

If we require in addition [6],[7], that the quantity

$$
\eta=c^{2}(m-M)
$$

may take any value, however, finite, as $c \rightarrow \infty$, then $m=M\left(1+O\left(1 / c^{2}\right)\right)$

(i.e., the "mass-shell limit"), and the relation between $E$ and $m$ takes on the form $[7]$

$$
E=m+\frac{\mathbf{p}^{2}}{2 M}
$$

where the Galilean mass $M$ coincides with the particle's intrinsic parameter.

In case of an equilibrium relativistic ensemble of distinguishable events such a transformation of the relativistic relation between $E$ and $m$ gives rise to the usual nonrelativistic Maxwell-Boltzmann distribution of $\mathbf{p}^{2} / 2 M[7]$.

In this work, we show that in the mass-shell limit, the equilibrium relativistic distribution for indistinguishable events used in ref. [1] approaches the distribution 
found by Jüttner and Synge [8] within the framework of an on-mass-shell relativistic kinetic theory. We also study the $c \rightarrow \infty$ limit of the on-mass-shell theory, and show that it goes smoothly to the Galilean form.

\section{Equilibrium relativistic distribution close to mass shell}

Consider the equilibrium relativistic distribution for indistinguishable events used in ref. [1] (we use the metric $g^{\mu \nu}=(-,+,+,+)$ and $q \equiv q^{\mu}, p \equiv p^{\mu}$, and assume no degeneracy; in the case of degeneracy all the corresponding formulas throughout the paper should be multiplied by degeneracy factor),

$$
f_{0}(q, p)=C(q) \frac{e^{A(q)\left(p+p_{c}\right)^{2}+B(q)}}{1 \mp e^{A(q)\left(p+p_{c}\right)^{2}+B(q)}}, \quad A(q)>0,
$$

which is normalized as

$$
\int d^{4} p f_{0}(q, p)=n(q)
$$

where $n(q)$ is the total number of events per unit space-time volume in the neighborhood of the point $q$.

It follows from the relations [7] (we suppress $c$ for the present consideration)

$$
\eta=m-M, \quad-\triangle \leq \eta \leq \triangle
$$

that

$$
M-\triangle \leq m \leq M+\triangle .
$$

Since $\triangle$ may take any value as small as one wishes, [] but not zero, i.e., the variation in mass of the particles of the ensemble may be very small, we can take the value of $p^{2} \equiv-m^{2}$ restricted to a small neighborhood of a fixed value $-M^{2}$. This permits us to write (4) as 9]

$$
f_{0}(q, p) \cong C(q) \frac{e^{-A\left(M^{2}+m_{c}^{2}\right)+B} e^{2 A p^{\mu} p_{c \mu}}}{1 \mp e^{-A\left(M^{2}+m_{c}^{2}\right)+B} e^{2 A p^{\mu} p_{c \mu}}} .
$$

Introducing hyperbolic variables [3] and performing integration [10], we obtain from (5) and (7) the normalization relation

$$
n(q)=C(q) \frac{4 \pi \triangle M^{2}}{A m_{c}} \sum_{n=1}^{\infty} \frac{( \pm 1)^{n+1}}{n} e^{-n A\left(M^{2}+m_{c}^{2}\right)+n B} K_{1}\left(2 n A M m_{c}\right),
$$

\footnotetext{
${ }^{1}$ It corresponds to the approximate mass shell condition $|m-M| \leq \triangle$.
} 
where $K_{1}$ is a Bessel function of the third kind, defined by

$$
K_{\nu}(z)=\frac{\pi i}{2} e^{\pi i \nu / 2} H_{\nu}^{(1)}(i z) .
$$

The average values of $p^{\mu}, p^{\mu} p^{\nu}$, etc., can be obtained from the corresponding relations of ref. [1], using the formula [1]

$$
K_{n}^{\prime}(z)=-K_{n+1}(z)+\frac{n}{z} K_{n}(z) .
$$

Thus

$$
\begin{aligned}
\left\langle p^{\mu}\right\rangle_{q}= & p_{c}^{\mu} \frac{M}{m_{c}} \frac{\sum_{n=1}^{\infty}\left\{( \pm 1)^{n+1} e^{-n A\left(M^{2}+m_{c}^{2}\right)+n B} K_{2}\left(2 n A M m_{c}\right) / n\right\}}{\sum_{n=1}^{\infty}\left\{( \pm 1)^{n+1} e^{-n A\left(M^{2}+m_{c}^{2}\right)+n B} K_{1}\left(2 n A M m_{c}\right) / n\right\}}, \\
\left\langle p^{\mu} p^{\nu}\right\rangle_{q}= & g^{\mu \nu} \frac{M}{2 A m_{c}} \frac{\sum_{n=1}^{\infty}\left\{( \pm 1)^{n+1} e^{-n A\left(M^{2}+m_{c}^{2}\right)+n B} K_{2}\left(2 n A M m_{c}\right) / n^{2}\right\}}{\sum_{n=1}^{\infty}\left\{( \pm 1)^{n+1} e^{-n A\left(M^{2}+m_{c}^{2}\right)+n B} K_{1}\left(2 n A M m_{c}\right) / n\right\}} \\
& +M^{2} \frac{p_{c}^{\mu} p_{c}^{\nu}}{m_{c}^{2}} \frac{\sum_{n=1}^{\infty}\left\{( \pm 1)^{n+1} e^{-n A\left(M^{2}+m_{c}^{2}\right)+n B} K_{3}\left(2 n A M m_{c}\right) / n\right\}}{\sum_{n=1}^{\infty}\left\{( \pm 1)^{n+1} e^{-n A\left(M^{2}+m_{c}^{2}\right)+n B} K_{1}\left(2 n A M m_{c}\right) / n\right\}} .
\end{aligned}
$$

Identifying, as in [1]]

$$
\begin{aligned}
2 A m_{c} & =\frac{1}{k_{B} T}, \\
m_{c} & =\frac{M}{\mu_{K}}, \\
B & =\frac{1}{k_{B} T}\left(\mu+\frac{m_{c}}{2}\right),
\end{aligned}
$$

where $T, \mu$ and $\mu_{K}$ are absolute temperature, chemical and mass potentials, respectively, we have

$$
\begin{aligned}
\left\langle p^{\mu}\right\rangle= & p_{c}^{\mu} \frac{M}{m_{c}} \frac{\sum_{n=1}^{\infty}\left\{( \pm 1)^{n+1} e^{\frac{n \mu^{\prime}}{k_{B} T}} K_{2}\left(\frac{n M}{k_{B} T}\right) / n\right\}}{\sum_{n=1}^{\infty}\left\{( \pm 1)^{n+1} e^{\frac{n \mu^{\prime}}{k_{B} T}} K_{1}\left(\frac{n M}{k_{B} T}\right) / n\right\}}, \\
\left\langle p^{\mu} p^{\nu}\right\rangle= & g^{\mu \nu} M k_{B} T \frac{\sum_{n=1}^{\infty}\left\{( \pm 1)^{n+1} e^{\frac{n \mu^{\prime}}{k^{T} T}} K_{2}\left(\frac{n M}{k_{B} T}\right) / n^{2}\right\}}{\sum_{n=1}^{\infty}\left\{( \pm 1)^{n+1} e^{\frac{n \mu^{\prime}}{k_{B} T}} K_{1}\left(\frac{n M}{k_{B} T}\right) / n\right\}} \\
& +M^{2} \frac{p_{c}^{\mu} p_{c}^{\nu}}{m_{c}^{2}} \frac{\sum_{n=1}^{\infty}\left\{( \pm 1)^{n+1} e^{\frac{n \mu^{\prime}}{k_{B} T}} K_{3}\left(\frac{n M}{k_{B} T}\right) / n\right\}}{\sum_{n=1}^{\infty}\left\{( \pm 1)^{n+1} e^{\frac{n \mu^{\prime}}{k_{B} T}} K_{1}\left(\frac{n M}{k_{B} T}\right) / n\right\}}
\end{aligned}
$$

\footnotetext{
${ }^{2}$ The first relation implies that in thermal equilibrium $A m_{c}$ is independent of $q$.
} 
where $\mu^{\prime} \equiv \mu-\frac{\mu_{K} M}{2 N}$ ( $N$ being the total number of events) is the "reduced" chemical potential approaching $\mu$ as $N \rightarrow \infty$ or $T \rightarrow 0$ (since in the latter case $\mu_{K} \rightarrow 0$ [1]).

As in previous works $[1],[3],[4]$, to obtain the local energy density we make a Lorentz transformation to the rest frame of the local average motion. According to (14), the relative velocity of the new frame is

$$
\mathbf{u}=\frac{\mathbf{p}_{\mathbf{c}}}{m_{c}}
$$

The rest frame energy is then

$$
\left\langle E^{\prime}\right\rangle=\frac{\langle E\rangle-\mathbf{u} \cdot \mathbf{p}}{\sqrt{1-\mathbf{u}^{2}}}
$$

so that

$$
\left\langle E^{\prime}\right\rangle=M \frac{\sum_{n=1}^{\infty}\left\{( \pm 1)^{n+1} e^{\frac{n \mu^{\prime}}{k_{B} T}} K_{2}\left(\frac{n M}{k_{B} T}\right) / n\right\}}{\sum_{n=1}^{\infty}\left\{( \pm 1)^{n+1} e^{\frac{n \mu^{\prime}}{k_{B} T}} K_{1}\left(\frac{n M}{k_{B} T}\right) / n\right\}} .
$$

To obtain the pressure and the energy density in our ensemble, as in previous works $[1],[3],[4]$, we study the particle energy-momentum tensor defined by the $R^{4}$ density,

$$
T^{\mu \nu}(q)=\sum_{i} \int d \tau \frac{p_{i}^{\mu} p_{i}^{\nu}}{M} \delta^{4}\left(q-q_{i}(\tau)\right)
$$

Using the result of $[3]$

$$
\left\langle T^{\mu \nu}(q)\right\rangle_{q}=T_{\triangle V} \int d^{4} p f_{0}(q, p) \frac{p^{\mu} p^{\nu}}{M}
$$

and the expression (15) for $\left\langle p^{\mu} p^{\nu}\right\rangle_{q}$, we obtain

$$
\begin{aligned}
\left\langle T^{\mu \nu}(q)\right\rangle_{q}= & \frac{T_{\triangle V} n(q)}{M}\left[g^{\mu \nu} \frac{M}{2 A m_{c}} \frac{\sum_{n=1}^{\infty}\left\{( \pm 1)^{n+1} e^{-n A\left(M^{2}+m_{c}^{2}\right)+n B} K_{2}\left(2 n A M m_{c}\right) / n^{2}\right\}}{\sum_{n=1}^{\infty}\left\{( \pm 1)^{n+1} e^{-n A\left(M^{2}+m_{c}^{2}\right)+n B} K_{1}\left(2 n A M m_{c}\right) / n\right\}}\right. \\
& \left.+M^{2} \frac{p_{c}^{\mu} p_{c}^{\nu}}{m_{c}^{2}} \frac{\sum_{n=1}^{\infty}\left\{( \pm 1)^{n+1} e^{-n A\left(M^{2}+m_{c}^{2}\right)+n B} K_{3}\left(2 n A M m_{c}\right) / n\right\}}{\sum_{n=1}^{\infty}\left\{( \pm 1)^{n+1} e^{-n A\left(M^{2}+m_{c}^{2}\right)+n B} K_{1}\left(2 n A M m_{c}\right) / n\right\}}\right]
\end{aligned}
$$

In this expression $T_{\triangle V}$ is the average passage interval in $\tau$ for the events which pass through a small (typical) four-volume $\triangle V$ in the neighborhood of the point $q$ of $R^{4}$.

The formula for the stress-energy tensor of a perfect fluid has the form [3]

$$
\left\langle T^{\mu \nu}(q)\right\rangle_{q}=p g^{\mu \nu}-(p+\rho) \frac{\left\langle p^{\mu}\right\rangle_{q}\left\langle p^{\nu}\right\rangle_{q}}{\left\langle p^{\lambda}\right\rangle_{q}\left\langle p_{\lambda}\right\rangle_{q}}
$$

where $p$ is the pressure and $\rho$ is the density of energy at $q$. 
According to (14),

$$
\frac{\left\langle p^{\mu}\right\rangle_{q}}{\sqrt{-\left\langle p^{\lambda}\right\rangle_{q}\left\langle p_{\lambda}\right\rangle_{q}}}=\frac{p_{c}^{\mu}}{m_{c}}
$$

hence

$$
p=\frac{T_{\triangle V} n(q)}{2 A m_{c}} \frac{\sum_{n=1}^{\infty}\left\{( \pm 1)^{n+1} e^{-n A\left(M^{2}+m_{c}^{2}\right)+n B} K_{2}\left(2 n A M m_{c}\right) / n^{2}\right\}}{\sum_{n=1}^{\infty}\left\{( \pm 1)^{n+1} e^{-n A\left(M^{2}+m_{c}^{2}\right)+n B} K_{1}\left(2 n A M m_{c}\right) / n\right\}}
$$

and

$$
p+\rho=T_{\triangle V} n(q) M \frac{\sum_{n=1}^{\infty}\left\{( \pm 1)^{n+1} e^{-n A\left(M^{2}+m_{c}^{2}\right)+n B} K_{3}\left(2 n A M m_{c}\right) / n\right\}}{\sum_{n=1}^{\infty}\left\{( \pm 1)^{n+1} e^{-n A\left(M^{2}+m_{c}^{2}\right)+n B} K_{1}\left(2 n A M m_{c}\right) / n\right\}} .
$$

To interpret these results, as in [1],[3],[4], we calculate the average (conserved) particle four-current having the microscopic form

$$
J^{\mu}(q)=\sum_{i} \int \frac{p_{i}^{\mu}}{M} \delta^{4}\left(q-q_{i}(\tau)\right) d \tau
$$

Using the result of [3]

$$
\left\langle J^{\mu}(q)\right\rangle_{q}=T_{\triangle V} \int d^{4} p \frac{p^{\mu}}{M} f_{0}(q, p)
$$

and expression (14) for $\left\langle p^{\mu}\right\rangle_{q}$, we obtain

$$
\left\langle J^{\mu}(q)\right\rangle_{q}=T_{\triangle V} n(q) \frac{p_{c}^{\mu}}{m_{c}} \frac{\sum_{n=1}^{\infty}\left\{( \pm 1)^{n+1} e^{-n A\left(M^{2}+m_{c}^{2}\right)+n B} K_{2}\left(2 n A M m_{c}\right) / n\right\}}{\sum_{n=1}^{\infty}\left\{( \pm 1)^{n+1} e^{-n A\left(M^{2}+m_{c}^{2}\right)+n B} K_{1}\left(2 n A M m_{c}\right) / n\right\}} .
$$

In the local rest frame $p_{c}^{\mu}=\left(m_{c}, \mathbf{0}\right)$,

$$
\left\langle J^{0}(q)\right\rangle_{q}=T_{\triangle V} n(q) \frac{\sum_{n=1}^{\infty}\left\{( \pm 1)^{n+1} e^{-n A\left(M^{2}+m_{c}^{2}\right)+n B} K_{2}\left(2 n A M m_{c}\right) / n\right\}}{\sum_{n=1}^{\infty}\left\{( \pm 1)^{n+1} e^{-n A\left(M^{2}+m_{c}^{2}\right)+n B} K_{1}\left(2 n A M m_{c}\right) / n\right\}} .
$$

Defining the density of particles per unit space volume as

$$
N_{0}(q)=\left\langle J^{0}(q)\right\rangle_{q},
$$

we obtain the relativistic equation of state

$$
\begin{aligned}
p & =\frac{N_{0}}{2 A m_{c}} \frac{\sum_{n=1}^{\infty}\left\{( \pm 1)^{n+1} e^{-n A\left(M^{2}+m_{c}^{2}\right)+n B} K_{2}\left(2 n A M m_{c}\right) / n^{2}\right\}}{\sum_{n=1}^{\infty}\left\{( \pm 1)^{n+1} e^{-n A\left(M^{2}+m_{c}^{2}\right)+n B} K_{2}\left(2 n A M m_{c}\right) / n\right\}} \\
& =N_{0} k_{B} T \frac{\sum_{n=1}^{\infty}\left\{( \pm 1)^{n+1} e^{\frac{n \mu^{\prime}}{k_{B} T}} K_{2}\left(\frac{n M}{k_{B} T}\right) / n^{2}\right\}}{\sum_{n=1}^{\infty}\left\{( \pm 1)^{n+1} e^{\frac{n \mu^{\prime}}{k_{B} T}} K_{2}\left(\frac{n M}{k_{B} T}\right) / n\right\}} .
\end{aligned}
$$


It follows from $(22),(23)$ that

$$
\frac{\rho}{p}=\frac{M}{k_{B} T} \frac{\sum_{n=1}^{\infty}\left\{( \pm 1)^{n+1} e^{\frac{n \mu^{\prime}}{k_{B} T}} K_{3}\left(\frac{n M}{k_{B} T}\right) / n\right\}}{\sum_{n=1}^{\infty}\left\{( \pm 1)^{n+1} e^{\frac{n \mu^{\prime}}{k_{B} T}} K_{2}\left(\frac{n M}{k_{B} T}\right) / n^{2}\right\}}-1 .
$$

Let us introduce $\Gamma$ through the relation

$$
p=(\Gamma-1) \rho .
$$

Then

$$
\Gamma=1+\frac{1}{\frac{M}{k_{B} T} \frac{\sum_{n=1}^{\infty}\left\{( \pm 1)^{n+1} e^{\frac{n \mu^{\prime}}{k_{B} T}} K_{3}\left(\frac{n M}{k_{B} T}\right) / n\right\}}{\sum_{n=1}^{\infty}\left\{( \pm 1)^{n+1} e^{\frac{n \mu^{\prime}}{k^{T}}} K_{2}\left(\frac{n M}{k_{B} T}\right) / n^{2}\right\}}-1} .
$$

Using the asymptotic formulas [12]

$$
K_{\nu}(z) \sim \begin{cases}\sqrt{\frac{\pi}{2 z}} e^{-z}, & z \rightarrow \infty \\ \frac{1}{2} \Gamma(\nu)\left(\frac{z}{2}\right)^{-\nu}, & z \rightarrow 0\end{cases}
$$

one obtains

$$
\Gamma=\left\{\begin{array}{l}
1, \quad T \rightarrow 0 \\
\frac{4}{3}, \quad T \rightarrow \infty
\end{array}\right.
$$

Since in thermal equilibrium there is no dependence on $q$, instead of $f_{0}(q, p)$ one can use equilibrium relativistic distribution function [5]

$$
f_{0}(p)=\frac{1}{e^{\left(E-\mu+\mu_{K} \frac{m^{2}}{2 M}\right) / k_{B} T} \mp 1}
$$

normalized ast

$$
\int \frac{d^{4} p}{(2 \pi)^{4}} f_{0}(p)=n
$$

where $n \equiv \frac{N}{V^{(4)}}$ is the event number density. In this case one obtains, instead of (8),

$$
n=\frac{\triangle M^{2} k_{B} T}{2 \pi^{3}} \sum_{n=1}^{\infty} \frac{( \pm 1)^{n+1}}{n} e^{\frac{n \mu^{\prime}}{k_{B} T}} K_{1}\left(\frac{n M}{k_{B} T}\right),
$$

and then, from $(22),(23),(27)$,

$$
p=\frac{\left(T_{\triangle V} \triangle\right) M^{2}\left(k_{B} T\right)^{2}}{2 \pi^{3}} \sum_{n=1}^{\infty} \frac{( \pm 1)^{n+1}}{n^{2}} e^{\frac{n \mu^{\prime}}{k_{B} T}} K_{2}\left(\frac{n M}{k_{B} T}\right),
$$

\footnotetext{
${ }^{3}$ This relation is helpful when studying an adiabatic equation of state 13.

${ }^{4}$ Eq. (36) is formally equivalent to $(4),(5)$, with $C(q)=\frac{1}{(2 \pi)^{4}}$.
} 


$$
\begin{gathered}
p+\rho=\frac{\left(T_{\triangle V} \triangle\right) M^{3} k_{B} T}{2 \pi^{3}} \sum_{n=1}^{\infty} \frac{( \pm 1)^{n+1}}{n} e^{\frac{n \mu}{k_{B} T}} K_{3}\left(\frac{n M}{k_{B} T}\right), \\
N_{0}=\frac{\left(T_{\triangle V} \triangle\right) M^{2} k_{B} T}{2 \pi^{3}} \sum_{n=1}^{\infty} \frac{( \pm 1)^{n+1}}{n} e^{\frac{n \mu^{\prime}}{k_{B} T}} K_{2}\left(\frac{n M}{k_{B} T}\right) .
\end{gathered}
$$

Using now the relation 14

$$
K_{n+1}(z)-K_{n-1}(z)=\frac{2 n}{z} K_{n}(z)
$$

one has from $(38),(39)$,

$$
\rho=3 p+\frac{\left(T_{\triangle V} \triangle\right) M^{3} k_{B} T}{2 \pi^{3}} \sum_{n=1}^{\infty} \frac{( \pm 1)^{n+1}}{n} e^{\frac{n \mu^{\prime}}{k_{B} T}} K_{1}\left(\frac{n M}{k_{B} T}\right) .
$$

The three relations $(38),(40),(42)$ coincide with the corresponding relations of an onmass-shell relativistic kinetic theory (see Appendix A), except for the additional factor $\left(T_{\triangle V} \triangle\right) / \pi$. Therefore, (both the on-shell and the off-shell in the limit $m^{2} \cong M^{2}$ ) approaches give the same results, if one takes $T_{\triangle V} \triangle=\pi$. Since $2 \triangle=\delta m$ is the width of the mass deviation from its sharp value $m^{2} \cong M^{2}$, the latter relation can be rewritten as

$$
T_{\triangle V} \delta m=2 \pi
$$

The presence of the factor $\left(T_{\triangle V} \triangle\right) / \pi$ in the expressions (38),(40) and (42) shows that, as $\delta m \rightarrow 0$, the corresponding quantities would go to zero, unless $T_{\triangle V} \rightarrow \infty$. Preservation of finite values of $p, \rho, N_{0}$ in this singular limit implies that the presence of events in the ensemble with very sharp mass requires the extension of the set of events over a very wide range of $\tau$, and therefore, $t$, since they are related through the equation of motion 15

$$
\frac{d t}{d \tau}=\frac{E}{M}
$$

hence

$$
\delta t=\langle d t\rangle=\langle d \tau\rangle \frac{\langle E\rangle}{M}=T_{\triangle V} \frac{\sum_{n=1}^{\infty}\left\{( \pm 1)^{n+1} e^{\frac{n \mu^{\prime}}{k_{B} T}} K_{2}\left(\frac{n M}{k_{B} T}\right) / n\right\}}{\sum_{n=1}^{\infty}\left\{( \pm 1)^{n+1} e^{\frac{n \mu^{\prime}}{k_{B} T}} K_{1}\left(\frac{n M}{k_{B} T}\right) / n\right\}},
$$

where we used the relation $\langle d \tau\rangle=T_{\triangle V}$ and the formula (16). In the Galilean limit $c \rightarrow \infty$ the argument of the $K$-functions goes to infinity?, so that one obtains, using the asymptotic formula (33) for $z \rightarrow \infty$,

$$
\langle d t\rangle=\langle d \tau\rangle
$$

in agreement with [6],[7].

\footnotetext{
${ }^{5}$ This argument is actually equal to $\frac{n M c^{2}}{k_{B} T}$.
} 
If $\delta m$ is not so small, the range of $\tau$, and therefore, $t$, need not be very large. In this case, for each value of the historical time parameter $\tau$, the set of events making up the ensemble may be concentrated in a range of $t$ which is not too large. The entire ensemble then moves in phase space with the development of $\tau$, and its stationary form corresponds to equilibrium. For $\delta m \rightarrow 0$ the ensemble fills a longer tube in phase space, and its displacement with $\tau$ is not as important. It is in this limit alone that a direct comparison with the usual (on-mass-shell) relativistic ensemble can be made, since in this case the system possesses a stationarity in space-time but not a non-trivial evolution in $\tau$, as was remarked in ref. [4].

\section{Limiting cases}

Now we wish to consider the expressions for $N_{0}, p$ and $\rho$ in the limiting cases $M \rightarrow 0$, $T \rightarrow \infty$, and $T \rightarrow 0$, and to compare them with the corresponding formulas of an on-mass-shell relativistic kinetic theory.

(i) $M \rightarrow 0$.

We remark that the dynamical evolution of the system contains the quantity $\frac{p^{\mu} p_{\mu}}{2 M}$ [15]. In the limit $M \rightarrow \infty$, this quantity is undefined; however, if we first go to the mass-shell limit, for which there is no non-trivial evolution in $\tau$, then this quantity becomes numerical-valued $(-M / 2)$ and the limit $M \rightarrow 0$ is well-defined. We therefore consider, for this limit, the on-shell results $(38),(40),(42)$, obtained from the distribution function (35).

Using the asymptotic formula (33) for $z \rightarrow 0$, one obtains the relations

$$
\begin{gathered}
N_{0}= \pm \frac{\left(T_{\triangle V} \triangle\right)\left(k_{B} T\right)^{3}}{\pi^{3}} L i_{3}\left( \pm e^{\frac{\mu^{\prime}}{k_{B} T}}\right) \\
p= \pm \frac{\left(T_{\triangle V} \triangle\right)\left(k_{B} T\right)^{4}}{\pi^{3}} L i_{4}\left( \pm e^{\frac{\mu^{\prime}}{k_{B} T}}\right) \\
\rho= \pm 3 \frac{\left(T_{\triangle V} \triangle\right)\left(k_{B} T\right)^{4}}{\pi^{3}} L i_{4}\left( \pm e^{\frac{\mu^{\prime}}{k_{B} T}}\right)
\end{gathered}
$$

(here $L i_{\nu}(z) \equiv \sum_{k=1}^{\infty} \frac{z^{k}}{k^{\nu}}$ is the so-called polylogarithm [16]), which coincide with the corresponding relations of an on-shell theory, if $T_{\triangle V} \triangle=\pi$ (see Appendix B).

(ii) $T \rightarrow \infty$.

This limit is mathematically equivalent to the limit $M \rightarrow 0$; physically it means that the particles become distinguishable and possess the relativistic distribution found by Synge [17]. Thus, one obtains the same expressions without summation on $k:$

$$
N_{0}=\frac{\left(T_{\triangle V} \triangle\right)\left(k_{B} T\right)^{3}}{\pi^{3}} e^{\frac{\mu^{\prime}}{k_{B} T}}
$$




$$
\begin{gathered}
p=\frac{\left(T_{\triangle V} \triangle\right)\left(k_{B} T\right)^{4}}{\pi^{3}} e^{\frac{\mu^{\prime}}{k_{B} T}}, \\
\rho=3 \frac{\left(T_{\triangle V} \triangle\right)\left(k_{B} T\right)^{4}}{\pi^{3}} e^{\frac{\mu^{\prime}}{k_{B} T}},
\end{gathered}
$$

in agreement with the textbook results, if $T_{\triangle V} \triangle=\pi[18]$.

(iii) $T \rightarrow 0$.

We study here the case in which the theory is on-mass-shell. If, in the limit $T \rightarrow 0$ we do not insist on the mass-shell constraint, then the mass distribution remains essentially relativistic. This case was treated for distinguishable events in ref. [7]. The indistinguishable case is treated in ref. [13].

Using the asymptotic formula (33) for $z \rightarrow \infty$, one obtains

$$
\begin{gathered}
N_{0}= \pm \frac{T_{\triangle V} \triangle}{\pi}\left(\frac{M k_{B} T}{2 \pi}\right)^{3 / 2} L i_{3 / 2}\left( \pm e^{\frac{\mu^{\prime}-M}{k_{B} T}}\right) \\
p= \pm \frac{T_{\triangle V} \triangle}{\pi}\left(\frac{M k_{B} T}{2 \pi}\right)^{3 / 2} k_{B} T L i_{5 / 2}\left( \pm e^{\frac{\mu^{\prime}-M}{k_{B} T}}\right)<<\rho \\
\rho= \pm \frac{T_{\triangle V} \triangle}{\pi}\left(\frac{M k_{B} T}{2 \pi}\right)^{3 / 2}\left[M L i_{3 / 2}\left( \pm e^{\frac{\mu^{\prime}-M}{k_{B} T}}\right)+3 k_{B} T L i_{5 / 2}\left( \pm e^{\frac{\mu^{\prime}-M}{k_{B} T}}\right)\right]=M N_{0}+3 p
\end{gathered}
$$

the formulas of a nonrelativistic statistical mechanics of indistinguishable particles if one takes $T_{\triangle V} \triangle=\pi$ and $\mu^{\prime}-M \cong \mu-M=\mu_{n r}$, the nonrelativistic chemical potential [19].

As was remarked in [7], the low-temperature limit does not necessarily coincide with the nonrelativistic limit of a theory (for example, very long wave length radiation in Maxwell's relativistic theory does not coincide with the static Coulomb limit; it is still radiation). We see, that the procedure of taking the limit $T \rightarrow 0$ on mass shell leads to a deformation of the symmetry group from Poincaré to Galilean invariance, which means that this procedure actually coincides with the Galilean limit $c \rightarrow \infty$ [21] (formally equivalent to the limit $T \rightarrow 0$, since in both cases the argument of the $K$-functions in the formulas (38),(40) and (42), $z \equiv \frac{n M c^{2}}{k_{B} T}$, goes to infinity). We shall return to a more detailed study of this point in a future research.

$$
\begin{aligned}
& { }^{6} \text { Indeed, using the formula (B.1) of Appendix B, one has }\left(\epsilon \equiv \frac{\mathbf{p}^{2}}{2 m}\right) \\
& N_{0}=\int \frac{d^{3} \mathbf{p}}{(2 \pi)^{3}} \frac{1}{e^{\left(\frac{\mathbf{p}^{2}}{2 m}-\mu\right) / k_{B} T} \mp 1}=\frac{m^{3 / 2}}{2^{1 / 2} \pi^{2}} \int \frac{\epsilon^{1 / 2} d \epsilon}{e^{(\epsilon-\mu) / k_{B} T} \mp 1}= \pm\left(\frac{m k_{B} T}{2 \pi}\right)^{3 / 2} L i_{3 / 2}\left( \pm e^{\frac{\mu}{k^{T} T}}\right),
\end{aligned}
$$

the normalization relation of a nonrelativistic statistical mechanics of indistinguishable particles, etc. 20.

${ }^{7}$ See the corresponding formulas of a nonrelativistic statistical mechanics of distinguishable particles in ref. [18], p.62, formulas (3.55). 


\section{Nonrelativistic distribution}

In conclusion we shall show that in the Galilean limit the variable $E-m=\frac{\mathbf{p}^{2}}{2 M}$ has the usual nonrelativistic distribution for indistinguishable particles.

We start with the normalization relation for the sharp-mass form $m^{2} \cong M^{2}$ of initial equilibrium relativistic distribution (4),

$$
n(q)=C(q) \int d^{4} p \frac{e^{-A M^{2}-A m_{c}^{2}+2 A p p_{c}+B}}{1 \mp e^{-A M^{2}-A m_{c}^{2}+2 A p p_{c}+B}} .
$$

This integral written in the local rest frame takes the form

$$
\int d E d^{3} \mathbf{p} \sum_{n=1}^{\infty}( \pm 1)^{n+1} e^{-2 n A m_{c} E} e^{-n\left(A M^{2}+A m_{c}^{2}-B\right)} \equiv \int d E d^{3} \mathbf{p} \sum_{n=1}^{\infty}( \pm 1)^{n+1} e^{-\frac{n E}{k_{B} T}} e^{\frac{n \mu^{\prime}}{k_{B} T}},
$$

or, in view of $(2),(3)$ and the relation $-\triangle \leq \eta \leq \triangle$,

$$
\sum_{n=1}^{\infty} \int_{M-\triangle}^{M+\triangle} d m e^{-\frac{n m}{k_{B} T}} \int d^{3} \mathbf{p} e^{-\frac{n}{k_{B} T}\left(\frac{\mathbf{p}^{2}}{2 M}-\mu^{\prime}\right)}
$$

Integration on $m$ gives

$$
\int_{M-\triangle}^{M+\triangle} d m e^{-\frac{n m}{k_{B} T}}=2 \triangle e^{-\frac{n M}{k_{B} T}}
$$

therefore

$$
n(q)=2 \triangle C(q) \int d^{3} \mathbf{p} \sum_{n=1}^{\infty}( \pm 1)^{n+1} e^{-\frac{n}{k_{B} T}\left(\frac{\mathbf{p}^{2}}{2 M}-\mu^{\prime}+M\right)} \equiv 2 \triangle C(q) \int d^{3} \mathbf{p} \frac{1}{e^{\left(\frac{\mathbf{p}^{2}}{2 M}-\tilde{\mu}\right) / k_{B} T} \mp 1},
$$

which is the usual (normalized) nonrelativistic Bose-Einstein/Fermi-Dirac distribution (we call $e \equiv \frac{\mathbf{p}^{2}}{2 M}$ )

$$
\begin{gathered}
f(e)=\tilde{D} \frac{e^{1 / 2}}{\exp \left(\frac{e-\tilde{\mu}}{k_{B} T} \mp 1\right.}, \\
\tilde{D}^{-1}=\Gamma\left(\frac{3}{2}\right)\left(k_{B} T\right)^{3 / 2} \sum_{n=1}^{\infty} \frac{( \pm 1)^{n+1}}{n^{3 / 2}} e^{\frac{n \tilde{\mu}}{k_{B} T}} .
\end{gathered}
$$

In this formulas

$$
\tilde{\mu}=\mu^{\prime}-M=\mu-M\left(1+\frac{\mu_{K}}{2 N}\right)
$$

is the "reduced" nonrelativistic chemical potential approaching, as $N \rightarrow \infty$ or $T \rightarrow 0$, the value $(\mu-M)[1],[19]$. 


\section{Concluding remarks}

We have considered the limiting mass-shell form of equilibrium relativistic distribution for indistinguishable events, studied previously in [1], and its Galilean limit. We have shown that the mass-shell forms of the basic thermodynamic quantities coincide with the corresponding expressions of a usual on-shell relativistic statistical mechanics, providing the important relation (43) for a width of the mass deviation over its sharp value. This relation implies that such a mass deviation in the system of events can be infinitely small. In this case the system of relativistic (mass shell) events becomes a stationary distribution in space-time, representing in this way the ensemble of the relativistic on-shell particles.

In ensembles, in which the appreciable mass fluctuations can occur, the relativistic distributions (the equilibrium solutions of the generalized Boltzmann equation) represent the corresponding equilibrium relativistic distributions of mass, considered previously in refs. [1],[4], for identical and nonidentical systems, respectively.

We have seen that the characteristic limiting cases give results in agreement with the corresponding limits of the usual theory; the low-temperature limit on mass shell in fact corresponds to the Galilean limit $c \rightarrow \infty$, in which the statistical mechanics of indistinguishable events goes over to the usual nonrelativistic statistical mechanics of indistinguishable particles.

For ensembles with mass fluctuations the framework of a manifestly covariant relativistic statistical mechanics provides corrections to results obtained within the usual on-shell theory. Physical consequences of the mass fluctuations for relativistic systems are considered in refs. [13], [24, 25], taking account anti-events, i.e., the events having the opposite sign of the mass potential $\mu_{K}$. In ref. [13] an adiabatic equation of state, $p \propto N_{0}^{6 / 5}$, is obtained for the system of degenerate off-shell fermions and possible implications in astrophysics are discussed. In 24 statistical mechanics of the bosonic event-anti-event system is considered. For such a system, at some critical temperature a special type of Bose-Einstein condensation sets in, which provides the events making up the ensemble a definite mass and represents, in this way, a phase transition to the usual on-shell sector. In ref. [25] possible applications of the off-shell theory in hadronic physics are discussed. The equation of state $p \propto T^{6}$, obtained in [25], corresponds to the "realistic" equation of state, proposed by Shuryak 26] for hot hadronic matter, which is in good agreement with experiment for the temperature range $0.2-1.0 \mathrm{GeV}$. 


\section{Appendix A}

Consider the following relation, which represents the normalization condition for the relativistic distribution function

$$
f_{0}(|\mathbf{p}|, m)=\frac{1}{e^{\left(\sqrt{\mathbf{p}^{2}+m^{2}}-\mu\right) / k_{B} T} \mp 1}
$$

$\left(\epsilon \equiv \sqrt{\mathbf{p}^{2}+m^{2}}\right):$

$$
N_{0}=\int \frac{d^{3} \mathbf{p}}{(2 \pi)^{3}} \frac{1}{e^{\left(\sqrt{\mathbf{p}^{2}+m^{2}}-\mu\right) / k_{B} T} \mp 1}=\frac{1}{2 \pi^{2}} \int_{m}^{\infty} d \epsilon \frac{e^{-(\epsilon-\mu) / k_{B} T} \epsilon \sqrt{\epsilon^{2}-m^{2}}}{1 \mp e^{-(\epsilon-\mu) / k_{B} T}} .
$$

Expanding the denominator into power series and changing variables $t=\epsilon / m$, one obtains

$$
N_{0}=\frac{m^{3}}{2 \pi^{2}} \sum_{n=1}^{\infty}( \pm 1)^{n+1} e^{\frac{n \mu}{k_{B} T}} \int_{1}^{\infty} d t t \sqrt{t^{2}-1} e^{-\frac{n m}{k_{B} T} t}
$$

Since [23]

$$
\int_{1}^{\infty} d t \sqrt{t^{2}-1} e^{-\frac{n m}{k_{B} T} t}=\frac{k_{B} T}{n m} K_{1}\left(\frac{n m}{k_{B} T}\right)
$$

one uses parametric differentiation with respect to $\left(n m / k_{B} T\right)$ and Eq. (10) of the main text to obtain

$$
\int_{1}^{\infty} d t t \sqrt{t^{2}-1} e^{-\frac{n m}{k_{B} T} t}=-\frac{\partial}{\partial\left(\frac{n m}{k_{B} T}\right)} \int_{1}^{\infty} d t \sqrt{t^{2}-1} e^{-\frac{n m}{k_{B} T} t}=\frac{k_{B} T}{n m} K_{2}\left(\frac{n m}{k_{B} T}\right) .
$$

Thus,

$$
N_{0}=\frac{m^{2} k_{B} T}{2 \pi^{2}} \sum_{n=1}^{\infty} \frac{( \pm 1)^{n+1}}{n} e^{\frac{n \mu}{k_{B} T}} K_{2}\left(\frac{n m}{k_{B} T}\right) .
$$

In the same way one can obtain 22]

$$
\begin{aligned}
& p=\int \frac{d^{3} \mathbf{p}}{(2 \pi)^{3}} \frac{\mathbf{p}^{2}}{3 \sqrt{\mathbf{p}^{2}+m^{2}}} \frac{1}{e^{\left(\sqrt{\mathbf{p}^{2}+m^{2}}-\mu\right) / k_{B} T} \mp 1}=\frac{m^{2}\left(k_{B} T\right)^{2}}{2 \pi^{2}} \sum_{n=1}^{\infty} \frac{( \pm 1)^{n+1}}{n^{2}} e^{\frac{n \mu}{k_{B} T}} K_{2}\left(\frac{n m}{k_{B} T}\right), \\
& \rho=\int \frac{d^{3} \mathbf{p}}{(2 \pi)^{3}} \frac{\sqrt{\mathbf{p}^{2}+m^{2}}}{e^{\left(\sqrt{\mathbf{p}^{2}+m^{2}}-\mu\right) / k_{B} T} \mp 1}=3 p+\frac{m^{3} k_{B} T}{2 \pi^{2}} \sum_{n=1}^{\infty} \frac{( \pm 1)^{n+1}}{n} e^{\frac{n \mu}{k_{B} T}} K_{1}\left(\frac{n m}{k_{B} T}\right) . \quad \text { (A.7) }
\end{aligned}
$$




\section{Appendix B}

First consider

$$
\begin{aligned}
\int_{0}^{\infty} \frac{a^{\nu} d a}{e^{a-b} \mp 1} & =\int_{0}^{\infty} d a a^{\nu} \frac{e^{-a+b}}{1 \mp e^{-a+b}}=\sum_{k=1}^{\infty}( \pm 1)^{k+1} e^{k b} \int_{0}^{\infty} d a a^{\nu} e^{-k a} \\
& =\Gamma(\nu+1) \sum_{k=1}^{\infty} \frac{( \pm 1)^{k+1}}{k^{\nu+1}} e^{k b}= \pm \Gamma(\nu+1) L i_{\nu+1}\left( \pm e^{b}\right)
\end{aligned}
$$

where $L i_{n}(z) \equiv \sum_{k=1}^{\infty} \frac{z^{k}}{k^{n}}$ is the polylogarithm [16]. Then

$$
\begin{gathered}
N_{0}=\int \frac{d^{3} \mathbf{p}}{(2 \pi)^{3}} \frac{1}{e^{(|\mathbf{p}|-\mu) / k_{B} T} \mp 1}=\frac{4 \pi}{(2 \pi)^{3}} \int \frac{p^{2} d p}{e^{(p-\mu) / k_{B} T} \mp 1}= \pm \frac{\left(k_{B} T\right)^{3}}{\pi^{2}} L i_{3}\left( \pm e^{\frac{\mu}{k_{B} T}}\right), \\
p=\int \frac{d^{3} \mathbf{p}}{(2 \pi)^{3}} \frac{\mathbf{p}^{2}}{3|\mathbf{p}|} \frac{1}{e^{(|\mathbf{p}|-\mu) / k_{B} T} \mp 1}=\frac{4 \pi}{3(2 \pi)^{3}} \int \frac{p^{3} d p}{e^{(p-\mu) / k_{B} T} \mp 1}= \pm \frac{\left(k_{B} T\right)^{4}}{\pi^{2}} L i_{4}\left( \pm e^{\frac{\mu}{k_{B} T}}\right), \\
\rho=\int \frac{d^{3} \mathbf{p}}{(2 \pi)^{3}} \frac{|\mathbf{p}|}{e^{(|\mathbf{p}|-\mu) / k_{B} T} \mp 1}=\frac{4 \pi}{(2 \pi)^{3}} \int \frac{p^{3} d p}{e^{(p-\mu) / k_{B} T} \mp 1}= \pm 3 \frac{\left(k_{B} T\right)^{4}}{\pi^{2}} L i_{4}\left( \pm e^{\frac{\mu}{k_{B} T}}\right) .
\end{gathered}
$$

\section{References}

[1] L. Burakovsky and L.P. Horwitz, Tel-Aviv Univ. preprint TAUP-2115-93

[2] L.P. Horwitz, W.C. Schieve and C. Piron, Ann. Phys. (N.Y.) 137 (1981) 306

[3] L.P. Horwitz, S. Shashoua and W.C. Schieve, Physica A 161 (1989) 300

[4] L. Burakovsky and L.P. Horwitz, Physica A 201 (1993) 666

[5] L. Burakovsky, Tel-Aviv Univ. preprint TAUP-2092-93

[6] L.P. Horwitz and F.C. Rotbart, Phys. Rev. D 24 (1981) 2127

[7] L. Burakovsky and L.P. Horwitz, Jour. Phys. A, in press

[8] S.R. de Groot, W.A. van Leeuwen and Ch.G. van Weert, Relativistic Kinetic Theory, (North-Holland, Amsterdam, 1980)

[9] F. Jüttner, Z. Phys. 47 (1928) 542

[10] I.S. Gradshteyn and I.M. Ryzhik, Tables of Integrals, Series, and Products (Academic Press, New York, 1980) p.358, subsection 3.547, formula 9 
[11] Ref. [9], p.970, subsection 8.486, formula 13

[12] M. Abramowitz and I.A. Stegun, Handbook of Mathematical Functions, p.375, (Dover, New York, 1970)

[13] L. Burakovsky, L.P. Horwitz and W.C. Schieve, Tel-Aviv Univ. preprint TAUP2124-93

[14] Ref. [9], p.970, subsection 8.486, formula 10

[15] L.P. Horwitz and C. Piron, Helv. Phys. Acta 46 (1973) 316

[16] A.P. Prudnikov et al. Integrals and Series, (Gordon and Breach, New York, 1980) Vol. 3, p.762, Appendix II.5

[17] J.L. Synge, The Relativistic Gas, (North-Holland, Amsterdam, 1957)

[18] See, for example, E.W. Kolb and M.S. Turner, The Early Universe, (Addison Wesley, New York, 1990) p.62, formulas (3.54)

[19] H.E. Haber and H.A. Weldon, Phys. Rev. Lett. 46 (1981) 1497; Phys. Rev. D 25 (1982) 502

[20] See, for example, K. Huang, Statistical Mechanics, (Wiley, New York, 1963)

[21] H.E. Haber and H.A. Weldon, J. Math. Phys. 23 (1982) 1852;

A. Actor, Nucl. Phys. B 256 (1986) 689

[22] Ref. [18], p.61, formulas $(3.47),(3,48)$

[23] Ref. [9], p.322, subsection 3.387, formula 3

[24] L. Burakovsky, L.P. Horwitz and W.C. Schieve, Tel-Aviv Univ. preprint TAUP2149-94

[25] L. Burakovsky and L.P. Horwitz, in preparation

[26] E.V. Shuryak, The QCD Vacuum, Hadrons and the Superdense Matter, (World Scientific, Singapore, 1988) 